\title{
The coexistence of a novel WNK1 variant and a copy number variation causes hereditary sensory and autonomic neuropathy type IIA
}

James Jiqi Wang ${ }^{1 \dagger}$, Bo Yu ${ }^{1+}$ and Zongzhe $\mathrm{Li}^{1,2^{*}}$ (D)

\begin{abstract}
Background: Hereditary sensory and autonomic neuropathy (HSAN) type II is a group of extremely rare autosomal recessive neurological disorders with heterogeneous clinical and genetic characteristics.

Methods: We performed high-depth next-generation targeted sequencing using a custom-ordered "HSAN" panel, covering WNK1, NTRK1, NGF, SPTLC1 and IKBKAP genes, to identify pathogenic variants of the proband as well as the family members. We also performed whole exome sequencing to further investigate the potential occurrence of additional pathogenic variants in genes that were not covered by the "HSAN" panel. Quantitative real-time PCR was used to identify pathogenic copy number variations (CNVs) and to analyze the mRNA level of WNK1 gene of the family. Western blot analysis was performed to evaluate the WNK1 protein expression level.

Results: After sequencing, a novel nonsense variant (c.2747 T > G, p.Leu916Ter) in exon 9 of WNK1 gene was identified in two patients (hemizygous) and their mother (heterozygous). This variant is absent in all public databases as well as in $600 \mathrm{Han}$ Chinese healthy controls. The region of this variant is evolutionary highly conserved. Furthermore, by quantitative real-time PCR using DNA of the pedigree, we revealed a large deletion containing the whole WNK1 gene in two patients. The WNK1 expression levels of the patients were significantly reduced.

Conclusions: Our study firstly revealed that the coexistence of a novel WNK1 nonsense variant and a CNV resulted in HSAN type IIA in a Han Chinese family.
\end{abstract}

Keywords: Hereditary sensory and autonomic neuropathies, HSAN IIA, WNK1, Targeted sequencing, Genetic diagnosis

\section{Background}

Hereditary sensory and autonomic neuropathies (HSAN), a group of extremely rare neurological diseases which are represented clinically and genetically heterogeneous, resulting in severe sensory dysfunction accompanied by variable degrees of autonomic dysfunction [1-4]. So far, mutations in at least 20 genes are

\footnotetext{
* Correspondence: marklee0208@163.com

† James Jiqi Wang and Bo Yu contributed equally to this work.

'Division of Cardiology, Departments of Internal Medicine and Genetic Diagnosis Center, Tongji Hospital, Tongji Medical College, Huazhong University of Science and Technology, Wuhan 430030, People's Republic of China

${ }^{2}$ Hubei Key Laboratory of Genetics and Molecular Mechanisms of Cardiological Disorders, Huazhong University of Science and Technology, Wuhan, China
}

known to be causative for HSAN with different inheritance patterns $[2,3,5-8]$.

HSAN type IIA (OMIM: 201300) is known as a subtype of HSAN type II $[1,9]$. The clinical features of this type including: early onset in childhood, chronic and recurrent ulcers at the tip of fingers and toes, unrecognized fractures of the feet/hands ("burning feet and hands"), anhidrosis, congenital insensitivity to pain $[9,10]$. The pathogenic variants in WNK1 gene are responsible for HSAN type IIA [9]. To date, only 11 pathogenic variants in WNK1 have been reported causing HSAN in the ClinVar database [11].

Copy number variations (CNVs) are large deletions/ duplications ranging in size from $50 \mathrm{bps}$ to megabases (Mbs) [12]. CNVs are known to cause 10 times more 
hereditable genetic differences compared with single nucleotide variants (SNV), and are associated with the pathogenesis of both Mendelian and complex diseases [13]. Recently, the combination of pathogenic SNV and $\mathrm{CNV}$ in one causative gene have been reported as a novel pathogenic mode in several autosomal recessive diseases $[14,15]$.

In this study, we firstly reported a Chinese HSAN type IIA family carrying a novel nonsense WNK1 variant, coupled with a large deletion containing the WNK1 gene.

\section{Methods}

\section{Next-generation sequencing}

Total DNA samples were extracted from peripheral blood of five family members (II-2, II-3, III-1, III-2, III-3) using a TIANamp Blood DNA Kit (TIANGEN). The extracted DNA samples were checked by electrophoresis to make sure it was without degradation and RNA pollution.

High depth parallel targeted next-generation semiconductor sequencing was performed on an Ion Proton sequencer (Life Technologies) using a custom-ordered "HSAN" panel. This panel contains all exons with flanking at least $5 \mathrm{bp}$ regulatory regions of five pathogenic HSAN genes (WNK1, NTRK1, NGF, SPTLC1, IKBKAP) (Additional file 1: Table S1) following the instructions as we previously described [16].

Whole-exome sequencing was performed with an Ion AmpliSeq ${ }^{\text {mix }}$ Exome Panel (Hi-Q) (57.7 Mb target region) on an Ion Proton sequencer (Life Technologies). In this way, we tried to analyze potential pathogenic variants in the targeted panel sequencing uncovered HSAN associated genes (KIF1A, FAM134B, FLVCR1 and SCN9A).

\section{Bioinformatic analysis}

The raw reads were aligned to the hg19/GRCh37 human reference genome by the Ion Torrent platform-specific software Torrent Suite v5.0.4. Only on-target reads were used to call variants using germline recommended parameters. Finally, all identified variants were annotated using the Ion Reporter ${ }^{\text {Tw }}$ software v5.0 (Life Technologies).

The ClinVar database (https://www.ncbi.nlm.nih.gov/ clinvar/) and the HGMD database (http://www.hgmd.cf. ac.uk/ac/search.php) were used to identify reported mutations. We firstly removed low-quality data (coverage less than 20-folds) and high-frequency variants (MAF more than $0.01 \%$ in the Exome Aggregation Consortium database) (http://exac.broadinstitute.org/). Then, the noncoding variants and synonymous variants were removed. Furthermore, the Polyphen-2 (score $>0.85$ ) (http://genetics.bwh.harvard.edu/pph2/) and the SIFT (score $<0.05$ ) (http://sift.jcvi.org) were used to evaluated the identified potential pathogenic missense variants.
The PhyloP scores (score $>0$ ) were used to assess the evolutionary conservation. Finally, the potential pathogenic variant was identified based on the recommendation of the American College of Medical Genetics and Genomics (ACMG) guideline for the interpretation of sequence variants criteria [12].

\section{Sanger sequencing}

We performed direct Sanger sequencing with specific primers in all identified potential pathogenic variants and low coverage regions ( $<20$-folds) using an Applied Biosystems 3500xl sequencer (Applied Biosystems). The PCR amplification and BigDye reaction were optimized using the Taq ${ }^{\text {Tx }}$ Hot Start enzyme (TaKaRa) and BigDye terminator cycle sequencing kit (Applied Biosystems), respectively. After validation in the patient, the potential pathogenic variants were also screened in the extra 600 unrelated Chinese healthy controls.

\section{Quantitative real-time PCR}

We performed copy number variations (CNVs) analysis of all sequenced subjects by using the CNV workflow on Ion Reporter ${ }^{\text {Tx }}$ software v5.0 (Life Technologies) to identify potential large deletion or insertion. We used a two-sample workflow (germline filter) to compare targeted sequencing reads of the test sample with a normal control sample. The control DNA sample was from a healthy person who performed CNV microarray (CytoScan HD, Affymetrix) previously and identified no CNV in our targeted sequencing regions. The identified potential pathogenic $\mathrm{CNV}$ was validated by quantitative real-time PCR analysis of gDNA using SYBR green and specific primers for WNK1 gene (F: 5'-CAGA GCCCTGGAATGAACTTG-3', R: 5' -TTAGGAGGGCT GCTTGTTGC-3'), with GAPDH as control gene (F: 5'-ATGCCTTCTTGCCTCTTGTCTC-3'， R: 5'-GTAA ACCTGGGGGAATACGTGA-3') on a StepOne Plus real-time PCR system (Applied Biosystems).

We also performed WNK1-mRNA expression analysis based on quantitative real-time PCR using specific primers (F: 5'-CCGCCACACTATGGACAAG-3', R: 5'-GGAGCACTCTGCGGGAC-3'), with GAPDH as housekeeping gene (F: 5'-TGCGCCTGCGGTAGAG C-3', R: 5'-CGGGCCAGCTAGCCTCGC-3'). Total RNAs were isolated from fresh leukocytes of the five family members (II-2, III-1, III-2, III-3, III-4) using TRIzol (Invitrogen) according to the manufacturer's instructions. The reverse-transcription was performed with M-MLV First-Strand cDNA Synthesis Kit (Invitrogen).

\section{WNK1 expression analysis}

Peripheral venous blood samples of four family members (II-2, III-1, III-2, III-3) were collected to extract leukocytes and to analyze the WNK1 protein expression levels 
using western blots as previously described [12]. Briefly, 2 $\mathrm{ml}$ whole blood for each person was treated with $4 \mathrm{ml}$ erythrocyte lysis buffer (TIANGEN). The collected leucocytes were homogenized in ice-cold lysis buffer. Lysates were separated by $6 \%$ SDS-PAGE, and transferred to $0.22 \mu \mathrm{m}$ PVDF membranes. The membranes were treated with 3\% BSA and 5\% non-fat milk for 2 hours to block. The primary antibodies (WNK1 polyclonal antibody, A2568, abclonal; GAPDH polyclonal antibody, AC001, abclonal) were used to incubate protein blots for $16 \mathrm{~h}\left(4^{\circ}\right.$ $\mathrm{C}$ overnight). Then, the protein blots were treated with peroxidase-conjugated secondary antibody (HRP goat anti-rabbit IgG, AS014, abclonal) for 2 hours. Finally, proteins were visualize using the enhanced chemiluminescence (BeyoECL plus, P0018, Beyotime).

\section{Statistical analysis}

All statistical analyses were conducted in SPSS software version 20.0. Person chi-Square tests were used to estimate differences between subjects. The two-sided $P$ value less than 0.05 was considered as statistically significant in this study.

\section{Results}

\section{Clinical features}

We studied two siblings (32-year-old male and 31-yeal-old female), offspring of non-consanguineous parents, featured with insensitivity to pain and sweating disorders since infancy (Fig. 1a). Neurologic examinations revealed the loss of pain sensation, diminished touch and temperature sense, and absent tendon reflexes. They had developed "burning feet and hands" resulted from continuous ulcers, infections and neurogenic acroosteolysis since their early childhood (Fig. 1b). Neurological exams of their mother (II-2) and sister (III-3) were normal. Their father (II-1), with nonspecific phenotype, died in car accident at the age of 45 .

\section{Sequencing output}

In the targeted next-generation sequencing of five members of the HSAN family, the average mapped reads was 290,144 per sample. On average, $97.66 \%$ of all 128 targeted-amplicons in the "HSAN" panel was sequenced at least 100 times and the base-read-depth was 1889 folds per sample. In the Whole exome sequencing of the two patients, the average mapped reads was 48,040,802. On average, $95.28 \%$ of target bases were covered more than 20 folds and the mean base-read-depth was 147 folds per sample.

\section{Pathogenic variant identification}

After bioinformatic analysis, a novel pathogenic nonsense variant (c.2747 T > G, p.Leu916Ter) in exon 9 of WNK1 gene was identified in three samples (II-2, III-1,

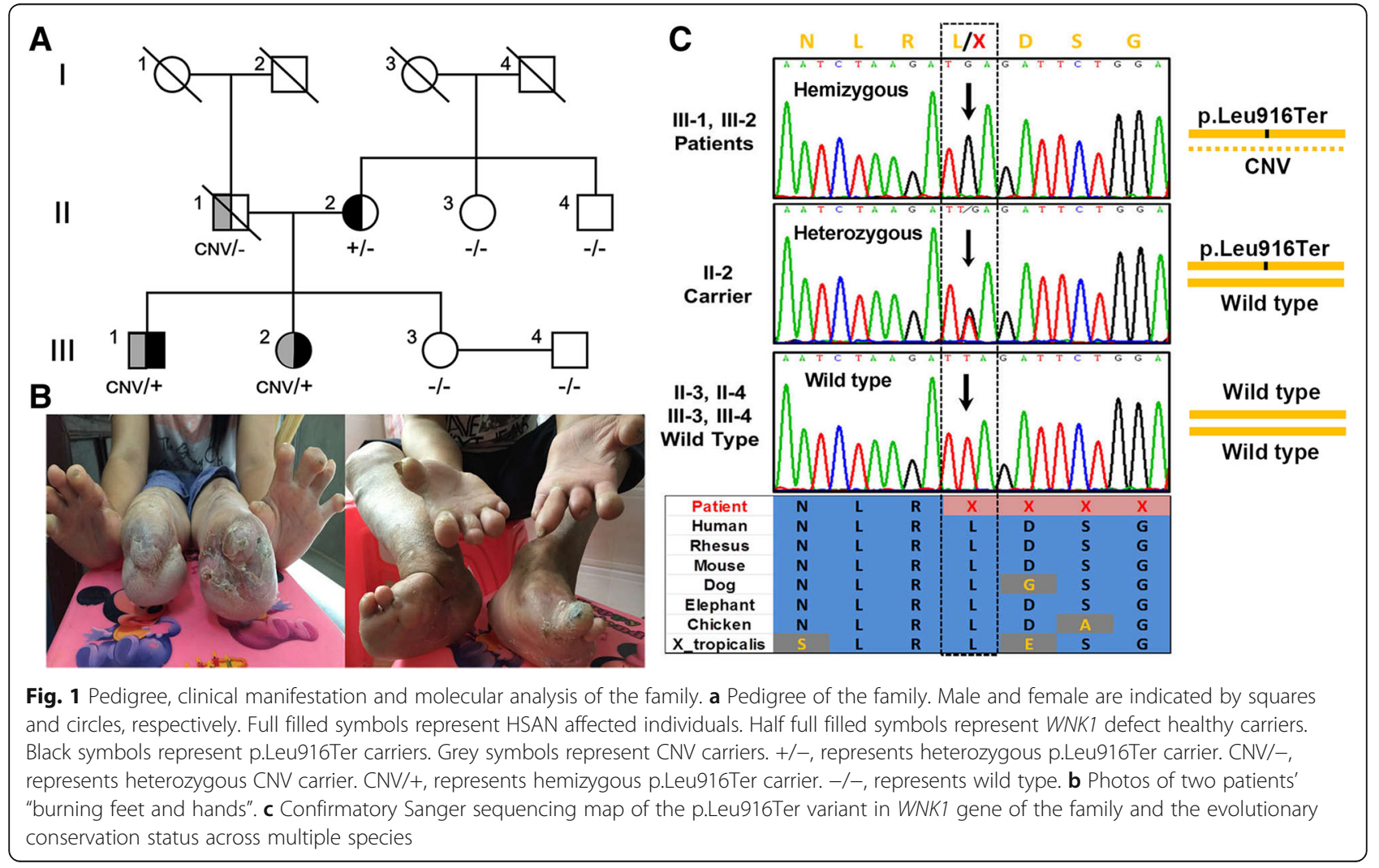


III-2). No extra potential pathogenic variants were identified in other HSAN-related genes, analyzed by whole-exome sequencing. Sanger sequencing demonstrated that the variant was "homozygous" (in fact was hemizygous) in two patients (III-1, III-2) and was heterozygous in the healthy mother (II-2). The pathogenic variant is located in an evolutionary highly conservative position across multiple species (Fig. 1c). This variant was predicted to introduce a premature stop codon and truncate the WNK1 protein. The novel nonsense variant (c.2747 T > G, p.Leu916Ter) is absent in the ClinVar database, the HGMD database, the ExAC database and the 1000-Genomes-Project database. Furthermore, it was not detected in 600 Han Chinese healthy controls.

\section{CNV identification}

We performed CNV analysis of all targeted genes based on the targeted sequencing raw data using the CNV plugin of Ion Reporter ${ }^{\mathrm{TM}}$ software v5.0. The CNV analysis suggested a heterozygous large deletion containing the entire WNK1 gene in two patients (III-1, III-2). In these two patients, all identified variants in WNK1 gene were hemizygous, which suggested a "loss of heterozygosity" phenomenon. The gDNA real-time PCR of the five participants (II-2, III-1, III-2, III-3, III-4) revealed that the
WNK1 DNA fold of two patients (III-1, III-2) was approximate half of the healthy families (Fig. 2a).

\section{Expression analysis of WNK1}

We performed quantitative real-time PCR to explore the effect of the identified pathogenic variant on mRNA amount of WNK1 using fresh isolated leucocytes of five family members (II-2, III-1, III-2, III-3, III-4). In the real-time PCR analysis of total RNA, the results revealed a decrease of the WNK1 mRNA of the patients (Fig. 2b).

We also performed western blots analysis using fresh isolated leucocytes of four family members (II-2, III-1, III-2, III-3) with different genotypes. The WNK1 protein expression level of the mother (II-2, heterozygous p.Leu916Ter carrier) was decreased to about half of the wild type daughter (III-3). The WNK1 protein expression levels of two patients (III-1 and III-2, hemizygous p.Leu916Ter carriers) were almost vanished (Fig. 2c).

\section{Discussion}

Our study reported a novel pathogenesis pattern of HSAN type IIA in a Han Chinese family. By targeted next-generation sequencing and quantitative real-time PCR, we identified a novel hemizygous pathogenic variant (c.2747 T > G, p.Leu916Ter) in WNK1 combined

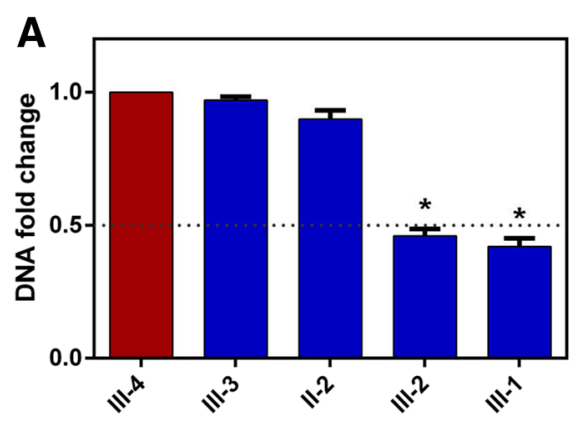

C

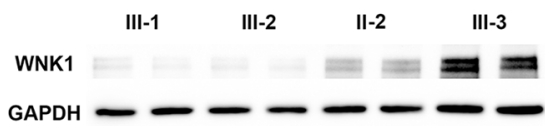

\section{B}

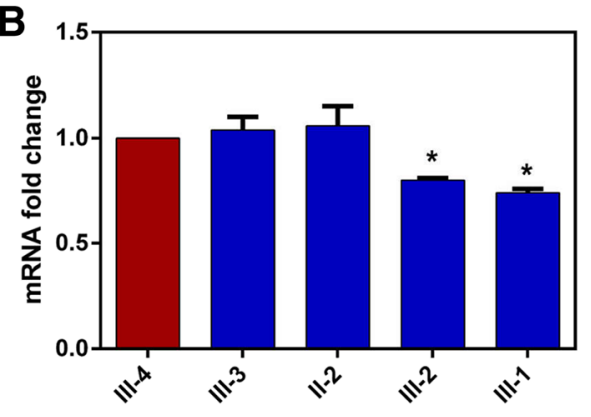

Relative WNK1 expression level

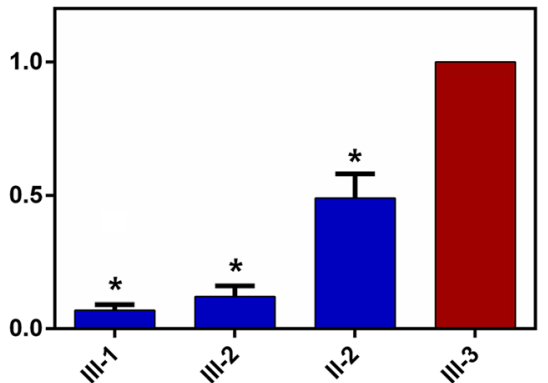

Fig. 2 Quantitative real-time PCR analysis of DNA and mRNA for WNK1 gene and transcript and Western Blot analysis of WNK1 protein. The WNK1 gene dosage was normalized using GAPDH gene as control. a The DNA fold change of the WNK1 gene for the family members. $\mathbf{b}$ The RNA fold change of the WNK1 gene for the family members. The WNK1 transcript amount was normalized using GAPDH as housekeeping gene. c The relative WNK1 protein expression level of the family members, using Western Blot. Protein expression was normalized by the use of the housekeeping GAPDH protein level. * indicates $p<0.05$ compared with wild type. The error bars refer to standard error (SE) of technical replicates 
with a large heterozygous deletion containing WNK1 (CNV) in the two patients with HSAN type IIA.

After targeted sequencing and Sanger sequencing, we found a novel "homozygous" nonsense WNK1 variants in two patients (III-1, III-2). The healthy mother (II-2) was heterozygous, which supported the autosomal recessive inheritance mode of HSAN type IIA. Unfortunately, the genotype of the healthy father (II-1) was unavailable because of car accident several years ago. After careful genetic counseling, however, we noted that there was no consanguineous relationship between the mother (II-2) and the father (II-1). Therefore, the chance that the father carried the same pathogenic variant was little. Two patients (III-1, III-2) carried the same "homozygous" nonsense variant, which implied that de novo inheritance mode was hardly to be the reason. From these points of view, we speculated that the father carried a heterozygous deletion involving the position of the novel identified nonsense variant.

By coverage analysis of next-generation sequencing reads and quantitative real-time PCR of DNA, we demonstrated a heterozygous large deletion containing the whole WNK1 gene in both patients (Fig. 2a). Therefore, the nonsense WNK1 pathogenic variant identified in the two patients by Sanger sequencing should be described as "hemizygous" variant. Since both of the affected siblings carried the large deletion (CNV), and although the DNA sample of the dead father was unavailable, we could speculate that the father, with nonspecific phenotype, was a heterozygous deletion carrier.. Although the DNA sample of the dead father was unavailable, we could speculate that the father was a heterozygous deletion carrier.

To further confirm our hypothesis and investigate the effect of CNV (haploinsufficiency) on WNK1 mRNA expression level, we performed quantitative real-time PCR to evaluate the WNK1 mRNA expression level of two patients (hemizygous), mother (heterozygous) and other healthy relatives (wild type). As is known, nonsense variants usually affect the expression of a gene during translation process (protein level). In contrast, copy number decrease could result in reduced expression of a gene since transcription (mRNA level). As we expected, there was no difference in the WNK1 mRNA level between the mother (heterozygous carrier of p.Leu916Ter) and the wild type. Hence, the p.Leu916Ter did not affect the expression of WNK1 in mRNA level. Notably, the WNK1 mRNA levels of the patients (carriers of CNV and p.Leu916Ter) were significantly decreased compared with the wild type. In western blot analysis, the relative WNK1 protein expression level of four family members with different genotypes (III-1, III-2: nonsense+CNV, hemizygous p.Leu916Ter; II-2: nonsense, heterozygous p.Leu916Ter; III-3: wild type) were compared. The
WNK1 protein expression level of II-2 (nonsense, heterozygous p.Leu916Ter) was approximate half of III-3 (wild type). The WNK1 protein expression levels of III-1 and III-2 (nonsense+CNV, hemizygous p.Leu916Ter) were almost vanished (Fig. 2c).

\section{Conclusion}

Our report firstly revealed a novel "hemizygous" pathogenesis mode (copy number decrease plus nonsense variant) of the HSAN type IIA and identified a novel nonsense pathogenic variant (c.2747 T > G, p.Leu916Ter). This study enriched the pathogenic mutation spectrum of HSAN and would facilitate the future genetic counseling.

\section{Additional file}

Additional file 1: Table S1. Sequened genes in the HSAN panel. (DOCX $14 \mathrm{~kb}$ )

\section{Abbreviations}

CNV: Copy number variation; HSAN: Hereditary sensory and autonomic neuropathy; MAF: Minor allele frequency

\section{Acknowledgments \\ The authors thank the family for their participation in this study. \\ Funding \\ This work was supported by grants from National Natural Science Foundation of China (81700413) and Integrated Innovative Team for Major Human Diseases Program of Tongji Medical College, HUST (2015ZDTD044). The funding body play no direct role in the design of the study, and collection, analysis, and interpretation of data, and in writing the manuscript. \\ Availability of data and materials \\ The datasets used and/or analyzed in this study are available from the corresponding author on reasonable request. \\ Authors' contributions \\ JJW performed the genomic DNA and RNA isolation, real-time PCR and Sanger sequencing, participated in the clinical samples and data collection. BY performed western blots and Whole exome sequencing. ZL performed the targeted sequencing, analyzed and interpreted the data, wrote the manuscript. All authors participated in the proofreading. All authors read and approved the final manuscript.}

\section{Ethics approval and consent to participate}

The publication of this manuscript has been reviewed and approved by the ethics committee of Tongji hospital, Tongji Medical College, Huazhong University of Science and Technology. We obtained fully informed written consents from all participants for participation in this study.

\section{Consent for publication}

We obtained fully informed written consents from all subjects for participation in this study and publication.

\section{Competing interests}

The authors declare that they have no competing interests.

\section{Publisher's Note}

Springer Nature remains neutral with regard to jurisdictional claims in published maps and institutional affiliations. 
Received: 24 February 2019 Accepted: 17 May 2019

Published online: 27 May 2019

\section{References}

1. Rotthier A, Baets J, De Vriendt E, Jacobs A, Auer-Grumbach M, Levy N, Bonello-Palot N, Kilic SS, Weis J, Nascimento A, et al. Genes for hereditary sensory and autonomic neuropathies: a genotype-phenotype correlation. Brain. 2009;132(Pt 10):2699-711.

2. Riviere JB, Ramalingam S, Lavastre V, Shekarabi M, Holbert S, Lafontaine J, Srour M, Merner N, Rochefort D, Hince P, et al. KIF1A, an axonal transporter of synaptic vesicles, is mutated in hereditary sensory and autonomic neuropathy type 2. Am J Hum Genet. 2011;89(2):219-30.

3. Yuan J, Matsuura E, Higuchi Y, Hashiguchi A, Nakamura T, Nozuma S, Sakiyama Y, Yoshimura A, Izumo S, Takashima H. Hereditary sensory and autonomic neuropathy type IID caused by an SCN9A mutation. Neurology. 2013;80(18):1641-9.

4. Carvalho OP, Thornton GK, Hertecant J, Houlden H, Nicholas AK, Cox JJ, Rielly M, Al-Gazali L, Woods CG. A novel NGF mutation clarifies the molecular mechanism and extends the phenotypic spectrum of the HSAN5 neuropathy. J Med Genet. 2011;48(2):131-5.

5. Yuan JH, Hashiguchi A, Yoshimura A, Sakai N, Takahashi MP, Ueda T, Taniguchi A, Okamoto S, Kanazawa N, Yamamoto Y, et al. WNK1/HSN2 founder mutation in patients with hereditary sensory and autonomic neuropathy: a Japanese cohort study. Clin Genet. 2017;92(6):659-63.

6. Davidson G, Murphy S, Polke J, Laura M, Salih M, Muntoni F, Blake J, Brandner S, Davies N, Horvath R, et al. Frequency of mutations in the genes associated with hereditary sensory and autonomic neuropathy in a UK cohort. J Neurol. 2012;259(8):1673-85.

7. Kurth I, Pamminger T, Hennings JC, Soehendra D, Huebner AK, Rotthier A, Baets J, Senderek J, Topaloglu H, Farrell SA, et al. Mutations in FAM134B, encoding a newly identified Golgi protein, cause severe sensory and autonomic neuropathy. Nat Genet. 2009;41(11):1179-81.

8. Chiabrando D, Castori M, di Rocco M, Ungelenk M, Giesselmann S, Di Capua M, Madeo A, Grammatico P, Bartsch S, Hubner CA, et al. Mutations in the Heme exporter FLVCR1 cause sensory neurodegeneration with loss of pain perception. PLoS Genet. 2016;12(12):e1006461.

9. Lafreniere RG, MacDonald ML, Dube MP, MacFarlane J, O'Driscoll M, Brais B, Meilleur S, Brinkman RR, Dadivas O, Pape T, et al. Identification of a novel gene (HSN2) causing hereditary sensory and autonomic neuropathy type ॥ through the study of Canadian genetic isolates. Am J Hum Genet. 2004; 74(5):1064-73.

10. Takagi M, Ozawa T, Hara K, Naruse S, Ishihara T, Shimbo J, Igarashi S, Tanaka K, Onodera O, Nishizawa M. New HSN2 mutation in Japanese patient with hereditary sensory and autonomic neuropathy type 2. Neurology. 2006; 66(8):1251-2.

11. Mroczek M, Kabzinska D, Kochanski A. Molecular pathogenesis, experimental therapy and genetic counseling in hereditary sensory neuropathies. Acta Neurobiol Exp (Wars). 2015;75(2):126-43.

12. Li Z, Chen P, Xu J, Yu B, Li X, Wang DW. A PLN nonsense variant causes severe dilated cardiomyopathy in a novel autosomal recessive inheritance mode. Int J Cardiol. 2019;279:122-5.

13. Pang AW, MacDonald JR, Pinto D, Wei J, Rafiq MA, Conrad DF, Park H, Hurles ME, Lee C, Venter JC, et al. Towards a comprehensive structural variation map of an individual human genome. Genome Biol. 2010; 11(5):R52.

14. Liu J, Zhou Y, Liu S, Song X, Yang XZ, Fan Y, Chen W, Akdemir ZC, Yan Z, Zuo $Y$, et al. The coexistence of copy number variations (CNVs) and single nucleotide polymorphisms (SNPs) at a locus can result in distorted calculations of the significance in associating SNPs to disease. Hum Genet. 2018;137(6-7):553-67.

15. Wu N, Ming X, Xiao J, Wu Z, Chen X, Shinawi M, Shen Y, Yu G, Liu J, Xie H et al. TBX6 null variants and a common hypomorphic allele in congenital scoliosis. N Engl J Med. 2015;372(4):341-50.

16. Li Z, Zhou C, Tan L, Chen P, Cao Y, Li C, Li X, Yan J, Zeng H, Wang DW, Wang DW. Sci China Life Sci. 2017;60(1):57-65.

\section{Ready to submit your research? Choose BMC and benefit from:}

- fast, convenient online submission

- thorough peer review by experienced researchers in your field

- rapid publication on acceptance

- support for research data, including large and complex data types

- gold Open Access which fosters wider collaboration and increased citations

- maximum visibility for your research: over $100 \mathrm{M}$ website views per year

At BMC, research is always in progress.

Learn more biomedcentral.com/submissions 